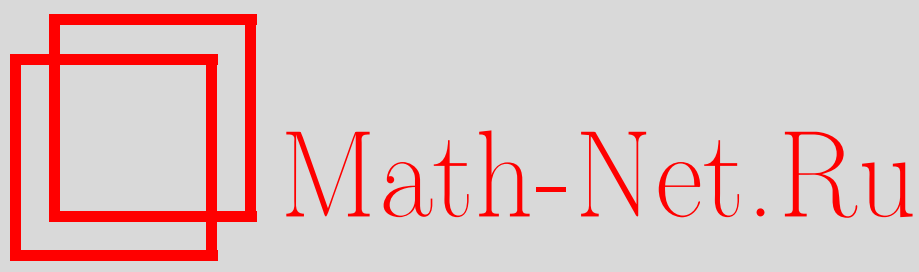

М. А. Ольшанецкий, В. К. Рогов, $q$-преобразование Фурье $q$-обобщенных функций, Матем. сб., 1999, том 190, номер 5, 93-112

DOI: https://doi.org/10.4213/sm403

Использование Общероссийского математического портала Math-Net.Ru подразумевает, что вы прочитали и согласны с пользовательским соглашением http://www . mathnet.ru/rus/agreement

Параметры загрузки:

IP: 34.229 .108 .108

26 апреля 2023 г., 15:59:08 


\title{
$q$-преобразование Фурье $q$-обобщенных функций
}

\begin{abstract}
Рассматриваются функции на решетке, порождаемой цельми степенями $q^{2}$, $0<q<1$. Строится $q$-аналог преобразования Фурье, базирующийся на интеграле Джексона в пространстве обобщенных функций на решетке.

Библиографияя: 8 названий.
\end{abstract}

\section{§1. Введение}

Классическое преобразование Фурье является мощным средством гармонического анализа на простых группах Ли и на однородных пространствах. В случае квантовых групп многие задачи могут быть просто решены с помощью преобразования Фурье в квантовых аффинных пространствах. Имеется ряд работ, посвященных квантовому преобразованию Фурье [1], [2], но их основной объект исследования - квантовая алгебра операторов без указания функциональных пространств, в которых эти операторы определены. Такой подход мешает применению полученных результатов в конкретных задачах гармонического анализа.

Здесь мы изучаем простейший одномерный случай, где весь эффект некоммутативности проявляется только в том, что функции заданы на решетке и очень проста алгебра, порожденная функциональными пространствами образов и прообразов. Эта ситуация может трактоваться как $q$-деформация классического преобразования Фурье, которой в последнее время уделяется особое внимание в силу ее связи с обобщениями классического гармонического осциллятора (см. [3]-[6]). В то время как одной из первых задач этих работ является изучение различных ядер $q$-преобразования Фурье, мы останавливаемся на функциональных пространствах и их $q$-экспоненциальном $q$-преобразовании Фурье. Наше исследование очень близко к классической ситуации, изученной в [7]. Мы рассматриваем пространства основных и $q^{2}$-обобшенных функций и определяем $q^{2}$-преобразование Фурье основных функций; $q^{2}$-преобразование Фурье $q^{2}$-обобшенных функций определяется с помощью равенства Парсеваля. В заключение мы приводим таблицу $q^{2}$-преобразований Фурье некоторых $q^{2}$-обобщенных функций.

Работа частично выполнена во время визита первого автора в Институт высших исследований (Bures-sur-Yvette). Он благодарен К. Гаведски за гостеприимство.

Работа первого автора частично поддержана Российской программой поддержки ведущих научных школ (грант № 96-15-96455), форондом INTAS (грант № 93-0166) и CRDF (грант № RM2-150).

Работа второго автора частично поддержана Российским фондом фундаментальных исследований (грант № 97-01-00747) и фондом НИОКР МПС РФ.

(C) М. А. ОльшАнецкий, В.-Б. К. РОГов 1999 


\section{§2. Некоторые предварительные соотношения}

Мы будем считать, что $z \in \mathbb{C}$ и $|q|<1$, если специально не оговорено противное.

Напомним некоторые обозначения [8]. Для любого $a$

$$
\begin{gathered}
(a, q)_{n}= \begin{cases}1 & \text { при } n=0, \\
(1-a)(1-a q) \cdots\left(1-a q^{n-1}\right) & \text { при } n \geqslant 1,\end{cases} \\
(a, q)_{\infty}=\lim _{n \rightarrow \infty}(a, q)_{n}, \\
{\left[\begin{array}{l}
l \\
i
\end{array}\right]_{q^{2}}=\frac{\left(q^{2}, q^{2}\right)_{l}}{\left(q^{2}, q^{2}\right)_{i}\left(q^{2}, q^{2}\right)_{l-i}} .}
\end{gathered}
$$

Рассмотрим $q^{2}$-экспоненты

$$
\begin{aligned}
& e_{q^{2}}(z)=\sum_{n=0}^{\infty} \frac{z^{n}}{\left(q^{2}, q^{2}\right)_{n}}=\frac{1}{\left(z, q^{2}\right)_{\infty}}, \quad|z|<1, \\
& E_{q^{2}}(z)=\sum_{n=0}^{\infty} \frac{q^{n(n-1)} z^{n}}{\left(q^{2}, q^{2}\right)_{n}}=\left(-z, q^{2}\right)_{\infty}
\end{aligned}
$$

и $q^{2}$-тригонометрические функции

$$
\begin{aligned}
& \cos _{q^{2}} z=\frac{1}{2}\left(e_{q^{2}}(i z)+e_{q^{2}}(-i z)\right), \quad \sin _{q^{2}} z=\frac{1}{2 i}\left(e_{q^{2}}(i z)-e_{q^{2}}(-i z)\right) \\
& \operatorname{Cos}_{q^{2}} z=\frac{1}{2}\left(E_{q^{2}}(i z)+E_{q^{2}}(-i z)\right), \quad \operatorname{Sin}_{q^{2}} z=\frac{1}{2 i}\left(E_{q^{2}}(i z)-E_{q^{2}}(-i z)\right)
\end{aligned}
$$

Мы рассмотрим также базисньй гипергеометрический ряд

$$
{ }_{0} \Phi_{1}\left(-; 0 ; q^{2}, z\right)=\sum_{n=0}^{\infty} \frac{q^{2 n(n-1)} z^{n}}{\left(q^{2}, q^{2}\right)_{n}} .
$$

Из формулы (2.1) следует, что $e_{q^{2}}(z)$ является мероморфной функцией с простьми полюсами в точках $z=q^{-2 k}, k=0,1, \ldots$

Лемма 2.1. Функиия $e_{q^{2}}(z)$ представляется в виде суммы простейших дробей:

$$
e_{q^{2}}(z)=\frac{1}{\left(q^{2}, q^{2}\right)_{\infty}} \sum_{k=0}^{\infty} \frac{(-1)^{k} q^{k(k+1)}}{\left(q^{2}, q^{2}\right)_{k}\left(1-z q^{2 k}\right)}
$$

ДокАЗАТЕЛЬСТво. Пусть

$$
e_{q^{2}}(z, n)=\frac{1}{\left(z, q^{2}\right)_{n}}=\sum_{k=0}^{n} \frac{c_{k, n}}{1-z q^{2 k}},
$$


где

$$
\begin{aligned}
c_{k, n} & =\operatorname{res}_{z=q^{-2 k}} e_{q^{2}}(z, n)=\lim _{z \rightarrow q^{-2 k}}\left(1-z q^{2 k}\right) e_{q^{2}}(z, n) \\
& =\frac{1}{\left(1-q^{-2 k}\right) \cdots\left(1-q^{-2}\right)\left(1-q^{2}\right) \cdots\left(1-q^{2(n-k)}\right)}=\frac{(-1)^{k} q^{k(k+1)}}{\left(q^{2}, q^{2}\right)_{k}\left(q^{2}, q^{2}\right)_{n-k}} .
\end{aligned}
$$

Следовательно,

$$
e_{q^{2}}(z, n)=\sum_{k=0}^{n} \frac{(-1)^{k} q^{k(k+1)}}{\left(q^{2}, q^{2}\right)_{k}\left(q^{2}, q^{2}\right)_{n-k}\left(1-z q^{2 k}\right)}
$$

Из неравенства

$$
\frac{1}{\left(q^{2}, q^{2}\right)_{n-k}}<\frac{1}{\left(q^{2}, q^{2}\right)_{\infty}}=e_{q^{2}}\left(q^{2}\right)
$$

мы получим

$$
e_{q^{2}}(z)=\lim _{n \rightarrow \infty} e_{q^{2}}(z, n)=\frac{1}{\left(q^{2}, q^{2}\right)_{\infty}} \sum_{k=0}^{\infty} \frac{(-1)^{k} q^{k(k+1)}}{\left(q^{2}, q^{2}\right)_{k}\left(1-z q^{2 k}\right)}
$$

Так как этот ряд абсолютно сходится при $q \in(0,1)$, представление $(2.6)$ справедливо для любого $z \neq q^{-2 k}$.

Из (2.3) мы получаем

СлЕДСТВИЕ 2.1. Функиии $\cos _{q^{2}} z u \sin _{q^{2}} z$ представляются в виде суммы простейших дробей:

$$
\begin{aligned}
& \cos _{q^{2}} z=\frac{1}{\left(q^{2}, q^{2}\right)_{\infty}} \sum_{k=0}^{\infty} \frac{(-1)^{k} q^{k(k+1)}}{\left(q^{2}, q^{2}\right)_{k}\left(1+z^{2} q^{4 k}\right)} \\
& \sin _{q^{2}} z=\frac{z}{\left(q^{2}, q^{2}\right)_{\infty}} \sum_{k=0}^{\infty} \frac{(-1)^{k} q^{k(k+3)}}{\left(q^{2}, q^{2}\right)_{k}\left(1+z^{2} q^{4 k}\right)} .
\end{aligned}
$$

ПРЕДЛОЖЕНИЕ 2.1. Если $z$ и q вещественные, то

$$
\begin{aligned}
& \left|\cos _{q^{2}} z\right| \leqslant \frac{\left(-q^{2}, q^{2}\right)_{\infty}}{\left(1+z^{2}\right)\left(q^{2}, q^{2}\right)_{\infty}} \\
& \left|\sin _{q^{2}} z\right| \leqslant \frac{|z|\left(-1, q^{2}\right)_{\infty}}{\left(1+z^{2}\right)\left(q^{2}, q^{2}\right)_{\infty}}
\end{aligned}
$$


ДокАЗАТЕльство. Так как

$$
\frac{1}{1+z^{2} q^{4 k}} \leqslant \frac{q^{-4 k}}{1+z^{2}}
$$

для любого вешественного $z$ и для любого $k \geqslant 0$, мы имеем

$$
\begin{aligned}
& \left|\sum_{k=0}^{\infty} \frac{(-1)^{k} q^{k(k+1)}}{\left(q^{2}, q^{2}\right)_{k}\left(1+z^{2} q^{4 k}\right)}\right| \leqslant \frac{1}{1+z^{2}} \sum_{k=0}^{\infty} \frac{q^{k(k-1)} q^{-2 k}}{\left(q^{2}, q^{2}\right)_{k}}=\frac{1}{1+z^{2}} E_{q^{2}}\left(q^{-2}\right), \\
& \left|\sum_{k=0}^{\infty} \frac{(-1)^{k} q^{k(k+3)}}{\left(q^{2}, q^{2}\right)_{k}\left(1+z^{2} q^{4 k}\right)}\right| \leqslant \frac{1}{1+z^{2}} \sum_{k=0}^{\infty} \frac{q^{k(k-1)}}{\left(q^{2}, q^{2}\right)_{k}}=\frac{1}{1+z^{2}} E_{q^{2}}(1) .
\end{aligned}
$$

Из последних соотношений и формул (2.7) и (2.8) вытекает утверждение предложения.

Следующее предложение сразу же получим из (2.2) и (2.4).

ПРЕДЛОЖЕНИЕ 2.2. Функщии $\operatorname{Cos}_{q^{2}} z u \operatorname{Sin}_{q^{2}} z$ удовлетворяют неравенст$в а \mathcal{M}$

$$
\left|\operatorname{Cos}_{q^{2}} z\right| \leqslant 1, \quad\left|\operatorname{Sin}_{q^{2}} z\right| \leqslant|z| .
$$

Рассмотрим тета-функцию

$$
\vartheta(u \mid \tau)=2 p^{1 / 8} \sin (\pi u) \prod_{n>0}\left(1-e^{2 \pi i u} p^{n}\right)\left(1-e^{-2 \pi i u} p^{n}\right)\left(1-p^{n}\right)
$$

где $p=\exp (2 \pi i \tau), \operatorname{Im} \tau>0$. Пусть

$$
\mathbf{Q}(z, q)=\left(1-q^{2}\right) \sum_{m=-\infty}^{\infty} \frac{1}{z q^{2 m}+z^{-1} q^{-2 m}}
$$

Тогда из модулярных свойств функции (2.10) следует, что

$$
\mathbf{Q}(z, q)=-\frac{1}{2 \pi i} \frac{\vartheta(u+\tau / 2 \mid \tau) \vartheta^{\prime}(0 \mid \tau)}{\vartheta(u \mid \tau) \vartheta(\tau / 2 \mid \tau)}
$$

где

$$
\tau=-\frac{2 i \ln q}{\pi}, \quad u=\frac{1}{\pi i} \ln z+\frac{1}{2} .
$$

Определим функцию

$$
\Theta(z)=\left(1-q^{2}\right) \sum_{m=-\infty}^{\infty} \sin _{q^{2}}\left(\left(1-q^{2}\right) q^{2 m} z\right)
$$


ПРЕДЛОЖЕНИЕ 2.3. Функиия $\Theta(z)$ обладает следующими свойствами:

1. $\Theta\left(q^{2 k} z\right)=\Theta(z)$ для любого $z \neq 0$,

2. $\Theta(z)=\mathbf{Q}\left(\left(1-q^{2}\right) z, q\right)$.

ДоКАЗАТЕЛьство. Свойство 1 сразу же следует из (2.11).

В силу формулы (2.8) функция $\Theta(z)$ может быть представлена в виде

$$
\Theta(z)=\frac{1-q^{2}}{\left(q^{2}, q^{2}\right)_{\infty}} \sum_{m=-\infty}^{\infty} q^{2 m} \sum_{k=0}^{\infty} \frac{(-1)^{k} q^{k(k+3)}\left(1-q^{2}\right) z}{\left(q^{2}, q^{2}\right)_{k}\left[1+\left(1-q^{2}\right)^{2} z^{2} q^{4(m+k)}\right]} .
$$

Так как внутренний ряд сходится равномерно по $m$, мы можем поменять порядок суммирования. Тогда, используя (2.1), (2.2) и (2.10), мы получим

$$
\begin{aligned}
\Theta(z) & =\frac{1-q^{2}}{\left(q^{2}, q^{2}\right)_{\infty}} \sum_{k=0}^{\infty} \frac{(-1)^{k} q^{k(k+3)}}{\left(q^{2}, q^{2}\right)_{k}} \sum_{m=-\infty}^{\infty} \frac{\left(1-q^{2}\right) z q^{2 m}}{1+\left(1-q^{2}\right)^{2} z^{2} q^{4(m+k)}} \\
& =\frac{1-q^{2}}{\left(q^{2}, q^{2}\right)_{\infty}} \sum_{k=0}^{\infty} \frac{(-1)^{k} q^{k(k+1)}}{\left(q^{2}, q^{2}\right)_{k}} \sum_{m=-\infty}^{\infty} \frac{\left(1-q^{2}\right) z q^{2 m}}{1+\left(1-q^{2}\right)^{2} z^{2} q^{4 m}}=\mathbf{Q}\left(\left(1-q^{2}\right) z, q\right) .
\end{aligned}
$$

Особо определим

$$
\Theta_{0}=\Theta(1)=\mathbf{Q}\left(1-q^{2}, q\right)
$$

ПРЕДЛОЖЕНИЕ 2.4. Для любого иелого $M>0$

$$
\begin{aligned}
& \left(1-q^{2}\right) z \sum_{m=-M}^{\infty} q^{2 m} \cos _{q^{2}}\left(\left(1-q^{2}\right) q^{2 m} z\right)=\sin _{q^{2}}\left(\left(1-q^{2}\right) q^{-2 M} z\right) \\
& \left(1-q^{2}\right) z \sum_{m=-M}^{\infty} q^{2 m} \sin _{q^{2}}\left(\left(1-q^{2}\right) q^{2 m} z\right)=1-\cos _{q^{2}}\left(\left(1-q^{2}\right) q^{-2 M} z\right)
\end{aligned}
$$

ДокАЗАТЕЛьСтво. Предположим, что $\left|\left(1-q^{2}\right) q^{-2 m} z\right|<1$. Тогда

$$
\begin{aligned}
(1- & \left.q^{2}\right) z \sum_{m=-M}^{\infty} q^{2 m} \cos _{q^{2}}\left(\left(1-q^{2}\right) q^{2 m} z\right) \\
& =\sum_{m=-M}^{\infty}\left(1-q^{2}\right) z q^{2 m} \sum_{k=0}^{\infty}(-1)^{k} \frac{\left(1-q^{2}\right)^{2 k} q^{4 m k} z^{2 k}}{\left(q^{2}, q^{2}\right)_{2 k}} \\
& =\sum_{k=0}^{\infty}(-1)^{k} \frac{\left(1-q^{2}\right)^{2 k+1} z^{2 k+1}}{\left(q^{2}, q^{2}\right)_{2 k}} \sum_{m=-M}^{\infty} q^{2 m(2 k+1)} \\
& =\sin _{q^{2}}\left(\left(1-q^{2}\right) q^{-2 M} z\right) .
\end{aligned}
$$

Для остальных значений $z \neq \pm i\left(1-q^{2}\right)^{-1} q^{-2 n}$ левая часть (2.13) определяется как аналитическое продолжение (2.15). Формула (2.14) доказывается аналогичньм образом. 


\section{§ 3. $q^{2}$-производная и $q^{2}$-интеграл}

Пусть $\mathscr{A}=C\left(z, z^{-1}\right)$ - алгебра формальных рядов Лорана. $q^{2}$-производная функции $f(z) \in \mathscr{A}$ определяется следующим образом:

$$
\partial_{z} f(z)=\frac{z^{-1}}{1-q^{2}}\left(f(z)-f\left(q^{2} z\right)\right)
$$

Для любого $n \geqslant 0$

$$
\partial_{z}^{k} z^{n}= \begin{cases}\frac{\left(q^{2}, q^{2}\right)_{n}}{\left(q^{2}, q^{2}\right)_{n-k}\left(1-q^{2}\right)^{k}} z^{n-k} & \text { при } 0 \leqslant k \leqslant n, \\ 0 & \text { при } k>n,\end{cases}
$$

и для любых $n \geqslant 0$ и $k \geqslant 0$

$$
\partial_{z}^{k} z^{-n-1}=(-1)^{k} q^{-k(2 n+k+1)} \frac{\left(q^{2}, q^{2}\right)_{n+k}}{\left(q^{2}, q^{2}\right)_{n}\left(1-q^{2}\right)^{k}} z^{-n-k-1} .
$$

$q^{2}$-интеграл (интеграл Джексона [8]) определяется как отображение $I_{q^{2}}$ алгебры $\mathscr{A}$ в пространство формальных числовых рядов:

$$
I_{q^{2}} f=\int d_{q^{2}} z f(z)=\left(1-q^{2}\right) \sum_{m=-\infty}^{\infty} q^{2 m}\left[f\left(q^{2 m}\right)+f\left(-q^{2 m}\right)\right] .
$$

ОПРЕДЕЛЕНИЕ 3.1. Функция $f(z)$ является локально $q^{2}$-интегрируемой, если $q^{2}$-интеграл

$$
\int_{a}^{b} d_{q^{2}} z f(z)=\left(1-q^{2}\right) \sum_{m=0}^{\infty} q^{2 m}\left[b f\left(b q^{2 m}\right)-a f\left(a q^{2 m}\right)\right]
$$

сушествует для любых конечных $a$ и $b$, т.е. ряд в правой части (3.5) сходится.

ОПРЕДЕЛЕНИЕ 3.2. Функция $f(z)$ является абсолютно $q^{2}$-интегрируемой, если ряд

$$
\sum_{m=-\infty}^{\infty} q^{2 m}\left[\left|f\left(q^{2 m}\right)\right|+\left|f\left(-q^{2 m}\right)\right|\right]
$$

сходится.

Пусть $\mathscr{B}$ - алгебра, аналогичная алгебре $\mathscr{A}$, но порождаемая образующими $s, s^{-1}$, которые связаны с $z$ коммутационным соотношением $z s=q^{2} s z$. Определим $q^{2}$-дифференцирование в $\mathscr{B}$

$$
\partial_{s} \phi(s)=\left(\phi(s)-\phi\left(q^{2} s\right)\right) \frac{s^{-1}}{1-q^{2}} .
$$

Обозначим через $\mathscr{A} \mathscr{B}$ алгебру с четырьмя образующими $z, z^{-1}, s, s^{-1}, q^{2}$-дифференцированием и с соотношениями

$$
z s=q^{2} s z, \quad \partial_{z} s=q^{-2} s \partial_{z}, \quad \partial_{s} z=q^{2} z \partial_{s}, \quad \partial_{z} \partial_{s}=q^{2} \partial_{s} \partial_{z}
$$


Мы будем рассматривать $\mathscr{A} \mathscr{B}$ как левый модуль по отношению к умножению на элементы из $\mathscr{A}$ и как правый модуль по отношению к умножению на элементы из $\mathscr{B}$.

Для определения $q^{2}$-интеграла в $\mathscr{A} \mathscr{B}$ мы упорядочим переменные интегрирования так, чтобы $z$ стояла слева, в то время как $s$ находилась бы справа. Например, если $f(z)=\sum_{r} a_{r} z^{r}$, то

$$
f(z s)=\sum_{r} a_{r}(z s)^{r}=\sum_{r} a_{r} q^{-r(r-1)} z^{r} s^{r} .
$$

Для удобства введем обозначение

$$
\ddagger g(z s) \ddagger=\sum_{r} a_{r} z^{r} s^{r}, \text { если } g(z)=\sum_{r} a_{r} z^{r} .
$$

Например, из (2.1), (2.2) и (3.6) мы можем получить

$$
E_{q^{2}}\left(i\left(1-q^{2}\right) z s\right)=\ddagger e_{q^{2}}\left(i\left(1-q^{2}\right) z s\right) \ddagger
$$

Следуя описанному вьше правилу, вычислим $q^{2}$-интегралы:

1. $\int d_{q^{2}} z z^{-1} E_{q^{2}}\left(i\left(1-q^{2}\right) z s\right)=2 i \Theta(s)$ (см. (2.12));

2. используя формулу (2.14) и предложение 2.1 , получим

$$
\int d_{q^{2}} z \Theta(z) E_{q^{2}}\left(i\left(1-q^{2}\right) z s\right)=2 i \Theta_{0} s^{-1}
$$

\section{§4. $q^{2}$-обобшенные функции}

Пусть $S_{q^{2}}=\{\phi(x)\}$ - пространство бесконечно $q^{2}$-дифференцируемых быстро убывающих функций, удовлетворяющих неравенству

$$
\left|x^{k} \partial_{x}^{l} \phi(x)\right| \leqslant C_{k, l}(q), \quad k \geqslant 0, \quad l \geqslant 0
$$

Пусть $S$ - пространство бесконечно дифференцируемых (в классическом смысле) быстро убывающих функций, удовлетворяющих неравенству

$$
\left|x^{k} \phi^{(l)}(x)\right| \leqslant C_{k, l}, \quad k \geqslant 0, \quad l \geqslant 0
$$

ПРЕДЛОЖЕНИЕ 4.1. Справедливо вложение $S \subset S_{q^{2}}$. 
ДокАЗАТЕльство. Обозначим через $\phi\left(x_{1}, x_{2}, \ldots, x_{k}\right)$ разделенную разность $k$-го порядка. Тогда нетрудно заметить, что для любого целого $l \geqslant 0$

$$
\phi\left(q^{2 l} x, q^{2 l-2} x, \ldots, x\right)=\frac{\left(q^{2}, q^{2}\right)_{l}}{\left(1-q^{2}\right)^{l}} \partial_{x}^{l} \phi(x) .
$$

С другой стороны, если $\phi(x) l$ раз дифференцируема (в классическом смысле), то существует такая точка $\xi \in\left(q^{2 l} x, x\right)$, что

$$
\phi\left(q^{2 l} x, q^{2 l-2} x, \ldots, x\right)=\frac{1}{l !} \phi^{(l)}(\xi) .
$$

Тогда из (4.3) и (4.4) вытекает, что

$$
\partial_{x}^{l} \phi(x)=\frac{\left(1-q^{2}\right)^{l}}{\left(q^{2}, q^{2}\right)_{l} l !} \phi^{(l)}(\xi), \quad \xi \in\left(q^{2 l} x, x\right)
$$

Следовательно, из (4.2)

$$
\left|x^{k} \partial_{x}^{l} \phi(x)\right| \leqslant \frac{\left(1-q^{2}\right)^{l}}{\left(q^{2}, q^{2}\right)_{l} l !} C_{k, l} .
$$

Наконец, если $\phi_{n}(x) \rightarrow 0$ при $n \rightarrow \infty$ в пространстве $S$, то $\phi_{n}(x) \rightarrow 0$ в пространстве $S_{q^{2}}$.

ОПРЕДЕЛЕНИЕ 4.1. Скелетом $\widehat{\phi}(z)$ функции $\phi(z) \in S_{q^{2}}$ назовем множество значений функции $\phi(z)$ на решетке, порождаемой целыми степенями $q^{2}$ :

$$
\widehat{\phi}(z)=\left.\phi(z)\right|_{z=q^{2 n}}, \quad n=0, \pm 1, \ldots
$$

Пространство скелетов мы будем обозначать через $\widehat{S}_{q^{2}}$. Функции

$$
\widehat{\phi}_{n}^{+}(z)=\left\{\begin{array}{ll}
1 & \text { при } z=q^{2 n}, \\
0 & \text { при } z \neq q^{2 n}
\end{array}, \quad \widehat{\phi}_{n}^{-}(z)= \begin{cases}1 & \text { при } z=-q^{2 n} \\
0 & \text { при } z \neq-q^{2 n}\end{cases}\right.
$$

образуют базис в пространстве $\widehat{S}_{q^{2}}$ :

$$
\widehat{\phi}(z)=\sum_{n=-\infty}^{\infty}\left[a_{n} \widehat{\phi}_{n}^{+}(z)+b_{n} \widehat{\phi}_{n}^{-}(z)\right], \quad a_{n}=\phi\left(q^{2 n}\right), \quad b_{n}=\phi\left(-q^{2 n}\right) .
$$

Здесь $\left|a_{n}\right|$ и $\left|b_{n}\right|$ ограничены при $n \rightarrow \infty$ и убывают быстрее любой степени $q^{-2 n}$ при $n \rightarrow-\infty$.

Топология в пространстве $\widehat{S}_{q^{2}}$ индуцируется топологией пространства $S_{q^{2}}(4.1)$ :

$$
\begin{array}{r}
\left|\frac{q^{2 n(k-l)}}{\left(1-q^{2}\right)^{l}} \sum_{i=1}^{l}(-1)^{i}\left[\begin{array}{l}
l \\
i
\end{array}\right]_{q^{2}} q^{-i(2 l-i-1)} a_{n+i}\right| \leqslant C_{k, l}(q), \\
\left|(-1)^{r-l} \frac{q^{2 n(k-l)}}{\left(1-q^{2}\right)^{l}} \sum_{i=1}^{l}(-1)^{i}\left[\begin{array}{l}
l \\
i
\end{array}\right]_{q^{2}} q^{-i(2 l-i-1)} b_{n+i}\right| \leqslant C_{k, l}(q) .
\end{array}
$$


Пусть $L_{q^{2}}$ - линейное отображение пространства $S_{q^{2}}$ в $\widehat{S}_{q^{2}}$, являющееся сужением функции на решетку

$$
L_{q^{2}} S_{q^{2}}=\widehat{S}_{q^{2}}
$$

Пусть $\Lambda \phi(z)=\phi\left(q^{2} z\right)$. Тогда

$$
\Lambda z=q^{2} z \Lambda, \quad \partial_{z} \Lambda=q^{2} \Lambda \partial_{z}
$$

Операторы $\Lambda$ и $\partial_{z}$ определены в $\widehat{S}_{q^{2}}$, так как

$$
\Lambda L_{q^{2}}=L_{q^{2}} \Lambda, \quad \partial_{z} L_{q^{2}}=L_{q^{2}} \partial_{z}
$$

Кроме того, $q^{2}$-интеграл от функций из $\operatorname{ker} L_{q^{2}}$ равен нулю и, следовательно, определен на факторпространстве

$$
\widehat{S}_{q^{2}}=S_{q^{2}} / \operatorname{ker} L_{q^{2}}
$$

В некоторых случаях мы будем под знаком $q^{2}$-интеграла записьвать элемент пространства $\widehat{S}_{q^{2}}$, имея в виду, что он является представителем класса из факторпространства.

ПРЕДЛОЖЕНИЕ 4.2. Если $\widehat{\phi}(z) \in \widehat{S}_{q^{2}}$, mo

$$
\int d_{q^{2}} z \partial_{z} \widehat{\phi}(z)=0
$$

ДоКАЗАТЕЛЬСТво. Из формул (3.1),(3.4) и (4.1) следует, что

$$
\begin{aligned}
\int d_{q^{2}} z \partial_{z} \widehat{\phi}(z) & =\sum_{m=-\infty}^{\infty}\left[\widehat{\phi}\left(q^{2 m}\right)-\widehat{\phi}\left(q^{2 m+2}\right)-\widehat{\phi}\left(-q^{2 m}\right)+\widehat{\phi}\left(-q^{2 m+2}\right)\right] \\
& =\lim _{M \rightarrow \infty}\left[\widehat{\phi}\left(q^{-2 M}\right)-\widehat{\phi}\left(-q^{-2 M}\right)\right]=0
\end{aligned}
$$

СлеДСтвиЕ 4.1 ( $q^{2}$-интегрирование по частям). Для любого $k \geqslant 0$

$$
\int d_{q^{2}} z \widehat{\phi}(z) \partial_{z}^{k} \widehat{\psi}(z)=(-1)^{k} q^{-k(k-1)} \int d_{q^{2}} z \partial_{z}^{k} \widehat{\phi}(z) \widehat{\psi}\left(q^{2 k} z\right)
$$

ОПРЕДЕЛЕНИЕ 4.2. $q^{2}$-обобщенной функиией $f$ над $\widehat{S}_{q^{2}}$ назовем линейный непрерывный функционал

$$
f: \widehat{S}_{q^{2}} \rightarrow \mathbb{C}
$$

Пространство $q^{2}$-обобщенных функций над $\widehat{S}_{q^{2}}$ обозначим через $\widehat{S}_{q^{2}}^{\prime}$. 
ОПРЕДЕЛЕНИЕ 4.3. Последовательность $\left\{f_{n}\right\}$ назовем сходящейся в $\widehat{S}_{q^{2}}^{\prime} \kappa f \in$ $\widehat{S}_{q^{2}}^{\prime}$, если для любой функции $\phi(z) \in \widehat{S}_{q^{2}}$ последовательность $\left\{\left\langle f_{n}, \phi\right\rangle\right\}$ сходится $\mathrm{K}\langle f, \phi\rangle$.

$q^{2}$-обобшенную функцию, определяемую $q^{2}$-интегралом

$$
\begin{aligned}
\langle f, \phi\rangle & =\int_{-\infty}^{\infty} d_{q^{2}} z \bar{f}(z) \phi(z) \\
& =\left(1-q^{2}\right) \sum_{m=-\infty}^{\infty} q^{2 m}\left[\bar{f}\left(q^{2 m}\right) \phi\left(q^{2 m}\right)+\bar{f}\left(-q^{2 m}\right) \phi\left(-q^{2 m}\right)\right]
\end{aligned}
$$

мы будем называть регулярной.

Предложение 4.2 и следствие 4.1 позволяют ввести $q^{2}$-дифференцирование в $\widehat{S}_{q^{2}}^{\prime}$ :

$$
\left\langle\partial_{z} f, \phi\right\rangle=-\left\langle\Lambda f, \partial_{z} \phi\right\rangle
$$

Приведем примеры $q^{2}$-обобщенных функций.

1. $\left\langle\theta_{q^{2}}^{+}, \phi\right\rangle=\int_{0}^{\infty} d_{q^{2}} z \widehat{\phi}(z)=\left(1-q^{2}\right) \sum_{m=-\infty}^{\infty} q^{2 m} \phi\left(q^{2 m}\right)$.

2. $\left\langle\theta_{q^{2}}^{-}, \phi\right\rangle=\int_{-\infty}^{0} d_{q^{2}} z \widehat{\phi}(z)=\left(1-q^{2}\right) \sum_{m=-\infty}^{\infty} q^{2 m} \phi\left(-q^{2 m}\right)$.

Таким образом, $q^{2}$-обобщенные функции $\theta_{q^{2}}^{+}$и $\theta_{q^{2}}^{-}$отвечают функциям

$$
\widehat{\theta}^{+}(z)=\sum_{n=-\infty}^{\infty} \widehat{\phi}_{n}^{+}(z), \quad \widehat{\theta}^{-}(z)=\sum_{n=-\infty}^{\infty} \widehat{\phi}_{n}^{-}(z) .
$$

3. $\left\langle\delta_{q^{2}}, \phi\right\rangle=\phi(0)=\lim _{m \rightarrow \infty} \frac{\phi\left(q^{2 m}\right)+\phi\left(-q^{2 m}\right)}{2}$.

При этом из (4.6), очевидно, следует:

$$
\begin{aligned}
& \left\langle\partial_{z}\left(\theta_{q^{2}}^{+}-\theta_{q^{2}}^{-}\right), \phi\right\rangle=-\left\langle\theta_{q^{2}}^{+}-\theta_{q^{2}}^{-}, \partial_{z} \phi\right\rangle \\
& =-\left(1-q^{2}\right) \sum_{m=-\infty}^{\infty} q^{2 m}\left(\frac{q^{-2 m}}{1-q^{2}}\left[\phi\left(q^{2 m}\right)-\phi\left(q^{2 m+2}\right)\right]\right. \\
& \left.\quad+\frac{q^{-2 m}}{1-q^{2}}\left[\phi\left(-q^{2 m}\right)-\phi\left(-q^{2 m+2}\right)\right]\right) \\
& =-\lim _{M \rightarrow \infty} \sum_{m=-M}^{M}\left[\phi\left(q^{2 m}\right)-\phi\left(q^{2 m+2}\right)+\phi\left(-q^{2 m}\right)-\phi\left(-q^{2 m+2}\right)\right] \\
& =-\lim _{M \rightarrow \infty}\left[\phi\left(q^{-2 M}\right)-\phi\left(q^{2 M+2}\right)+\phi\left(-q^{-2 M}\right)-\phi\left(-q^{2 M+2}\right)\right]=2 \phi(0),
\end{aligned}
$$

т.e.

$$
\partial_{z}\left(\theta_{q^{2}}^{+}(z)-\theta_{q^{2}}^{-}(z)\right)=2 \delta_{q^{2}}(z)
$$


4. $\left\langle z^{-1}, \phi\right\rangle=\int_{0}^{\infty} d_{q^{2}} z z^{-1}[\widehat{\phi}(z)-\widehat{\phi}(-z)]=\left(1-q^{2}\right) \sum_{m=-\infty}^{\infty}\left[\phi\left(q^{2 m}\right)-\phi\left(-q^{2 m}\right)\right]$.

5. Для любого $k \geqslant 0$

$$
\begin{aligned}
\left\langle z^{-k-1}, \phi\right\rangle & =(-1)^{k} q^{k(k+1)} \frac{\left(1-q^{2}\right)^{k}}{\left(q^{2}, q^{2}\right)_{k}}\left\langle\partial_{z}^{k} z^{-1}, \phi\right\rangle=\frac{\left(1-q^{2}\right)^{k}}{\left(q^{2}, q^{2}\right)_{k}}\left\langle z^{-1}, \partial_{z}^{k} \phi\right\rangle \\
& =\frac{\left(1-q^{2}\right)^{k+1}}{\left(q^{2}, q^{2}\right)_{k}} \sum_{m=-\infty}^{\infty}\left[\left.\partial_{z}^{k} \phi(z)\right|_{z=q^{2 m}}-\left.\partial_{z}^{k} \phi(z)\right|_{z=-q^{2 m}}\right]
\end{aligned}
$$

6. Для любого $\nu>-1$

$$
\left\langle z_{+}^{\nu}, \phi\right\rangle=\int_{0}^{\infty} d_{q^{2}} z z^{\nu} \widehat{\phi}(z)=\left(1-q^{2}\right) \sum_{m=-\infty}^{\infty} q^{2 m(\nu+1)} \phi\left(q^{2 m}\right)
$$

Так как для любого $k \geqslant 0$

$$
\partial_{z}^{k} z^{\nu}=(-1)^{k} q^{k(2 \nu-k+1)} \frac{\left(q^{-2 \nu}, q^{2}\right) k}{\left(1-q^{2}\right)^{k}} z^{\nu-k}
$$

TO

$$
\left\langle z_{+}^{\nu-k}, \phi\right\rangle=\left.\frac{\left(1-q^{2}\right)^{k+1}}{\left(q^{-2 \nu}, q^{2}\right)_{k}} \sum_{m=-\infty}^{\infty} q^{2 m(\nu+1)} \partial_{z}^{k} \phi(z)\right|_{z=q^{2 m}}
$$

7. Аналогично, для любого $\nu>-1$

$$
\left\langle z_{-}^{\nu}, \phi\right\rangle=\int_{-\infty}^{0} d_{q^{2}} z(-z)^{\nu} \widehat{\phi}(z)=\left(1-q^{2}\right) \sum_{m=-\infty}^{\infty} q^{2 m(\nu+1)} \phi\left(-q^{2 m}\right)
$$

и для любого $k \geqslant 0$

$$
\left\langle z_{-}^{\nu-k}, \phi\right\rangle=\left.(-1)^{k} \frac{\left(1-q^{2}\right)^{k+1}}{\left(q^{-2 \nu}, q^{2}\right)_{k}} \sum_{m=-\infty}^{\infty} q^{2 m(\nu+1)} \partial_{z}^{k} \phi(z)\right|_{z=-q^{2 m}}
$$

\section{§5. $q^{2}$-преобразование Фурье}

Рассмотрим пространство $S^{q^{2}}=\{\psi(s)\}$, содержашее функции того же класса, что и $S_{q^{2}}=\{\phi(z)\}(4.1)$, но $s$ и $z$ ведут себя как образуюшие алгебры $\mathscr{A} \mathscr{B}(3.6)$. Введем в $S^{q^{2}}$ такую же топологию, как и в $S_{q^{2}}$ :

$$
\left|s^{k} \partial_{s}^{l} \phi(s)\right| \leqslant C_{k, l}(q), \quad k \geqslant 0, \quad l \geqslant 0,
$$

в силу чего эти пространства становятся изоморфньми.

Определим отображение $S_{q^{2}}$ в $S^{q^{2}}$

$$
S_{q^{2}} \stackrel{L_{q^{2}}}{\longrightarrow} \widehat{S}_{q^{2}} \stackrel{\mathscr{F}_{q^{2}}}{\longrightarrow} S^{q^{2}}
$$


где

$$
\mathscr{F}_{q^{2}} \phi(z)=\int d_{q^{2}} z \phi(z){ }_{0} \Phi_{1}\left(-; 0 ; q^{2}, i\left(1-q^{2}\right) q^{2} z s\right)
$$

является $q^{2}$-преобразованием Фурье, а $0 \Phi_{1}$ определяется формулой (2.5).

Из (5.2) следует, что преобразование $\mathscr{F}_{q^{2}}$ определено на $S_{q^{2}}$, и в некоторых случаях мы будем оставлять то же обозначение $\mathscr{F} q^{2}$ для отображения из $S_{q^{2}}$ в $S^{q^{2}}$, опуская действие оператора $L_{q^{2}}$.

Нашей целью является построение отображения $\mathscr{F}_{q^{2}}^{-1}$, обратного к $\mathscr{F} q^{2}$, и доказательство их непрерывности. Точнее, мы определяем дуальное пространство скелетов $\widehat{S}^{q^{2}}$ для функций из $S^{q^{2}}$ и отображение

$$
\mathscr{F}_{q^{2}}^{-1} \psi(s)=\frac{1}{2 \Theta_{0}} \int E_{q^{2}}\left(-i\left(1-q^{2}\right) z s\right) \psi(s) d_{q^{2}} s, \quad \psi(s) \in \widehat{S}^{q^{2}},
$$

из $\widehat{S}^{q^{2}}$ в $S_{q^{2}}$.

Рассмотрим диаграмму

$$
\begin{aligned}
& S_{q^{2}} \stackrel{L_{q^{2}}}{\longrightarrow} \widehat{S}_{q^{2}} \\
& \left.\uparrow_{\mathscr{F}_{q^{2}}-1}\right\rfloor_{\mathscr{F}_{q^{2}}} \\
& \widehat{S}^{q^{2} \stackrel{L_{q^{2}}}{\longrightarrow}} S^{q^{2}} .
\end{aligned}
$$

Мы докажем

ПРеДЛОЖЕНИЕ 5.1. 1. Отображения

$$
\begin{gathered}
\mathscr{F}_{q^{2}} L_{q^{2}}: S_{q^{2}} \rightarrow S^{q^{2}}, \\
\mathscr{F}_{q^{2}}^{-1} L_{q^{2}}: S^{q^{2}} \rightarrow S_{q^{2}}
\end{gathered}
$$

являются изоморфизмами топологических пространств.

2. Отображения

$$
\begin{gathered}
L_{q^{2}} \mathscr{F}_{q^{2}}^{-1} L_{q^{2}} \mathscr{F}_{q^{2}}: \widehat{S}_{q^{2}} \rightarrow \widehat{S}_{q^{2}}, \\
L_{q^{2}} \mathscr{F}_{q^{2}} L_{q^{2}} \mathscr{F}_{q^{2}}^{-1}: \widehat{S}^{q^{2}} \rightarrow \widehat{S}^{q^{2}}
\end{gathered}
$$

яв.ляются тождественными в $\widehat{S}_{q^{2}}$ и в $\widehat{S}^{q^{2}}$ соответственно.

Мы начнем с доказательства вспомогательного утверждения.

Лемма 5.1. Справедливо равенство

$$
\int d_{q^{2}} z e_{q^{2}}\left(-i\left(1-q^{2}\right) z\right){ }_{0} \Phi_{1}\left(-; 0 ; q^{2}, i\left(1-q^{2}\right) q^{2} z s\right)= \begin{cases}\frac{2}{1-q^{2}} \Theta_{0} & \text { npu } s=1, \\ 0 & \text { npu } s \neq 1,\end{cases}
$$

әде $\Theta_{0}$ определяется формулой (2.12). 
ДокАЗАТЕльСтво. В соответствии с нашим определением $q^{2}$-интеграла переменные под знаком $q^{2}$-интеграла должны быть упорядочены. Из формул $(2.2),(2.5)$ и (3.6) следует, что

$$
{ }_{0} \Phi_{1}\left(-; 0 ; q^{2}, i\left(1-q^{2}\right) q^{2} z s\right)=\ddagger E_{q^{2}}\left(i\left(1-q^{2}\right) q^{2} z s\right) \ddagger,
$$

и мы приходим к $q^{2}$-интегралу

$$
\int d_{q^{2}} z e_{q^{2}}\left(-i\left(1-q^{2}\right) z\right) \ddagger E_{q^{2}}\left(i\left(1-q^{2}\right) q^{2} z s\right) \ddagger .
$$

Используя (2.1) и (2.2), получаем

$$
\begin{aligned}
\partial_{z}\left[e_{q^{2}}\left(-i\left(1-q^{2}\right) z\right) \ddagger E_{q^{2}}\right. & \left.\left(i\left(1-q^{2}\right) z s\right) \ddagger\right] \\
& =-i e_{q^{2}}\left(-i\left(1-q^{2}\right) z\right) \ddagger E_{q^{2}}\left(i\left(1-q^{2}\right) q^{2} z s\right) \ddagger(1-s) .
\end{aligned}
$$

Следовательно, если $s \neq 1$, то

$$
\begin{aligned}
\int d_{q^{2}} z e_{q^{2}}\left(-i\left(1-q^{2}\right) z\right) \ddagger E_{q^{2}}\left(i\left(1-q^{2}\right) q^{2} z s\right) \ddagger \\
\quad=i(1-s)^{-1} \int d_{q^{2}} z \partial_{z}\left[e_{q^{2}}\left(-i\left(1-q^{2}\right) z\right) \ddagger E_{q^{2}}\left(i\left(1-q^{2}\right) z s\right) \ddagger\right] .
\end{aligned}
$$

Последний $q^{2}$-интеграл может быть представлен в виде (см. (2.3) и (2.4))

$$
\begin{aligned}
(s-1)^{-1} \lim _{M \rightarrow \infty}\left[\cos _{q^{2}}(\right. & \left.\left(1-q^{2}\right) q^{-2 M}\right) \operatorname{Sin}_{q^{2}}\left(\left(1-q^{2}\right) q^{-2 M+2} s\right) \\
- & \sin _{q^{2}}\left(\left(1-q^{2}\right) q^{-2 M}\right) \operatorname{Cos}_{q^{2}}\left(\left(1-q^{2}\right) q^{-2 M+2} s\right) \\
- & \cos _{q^{2}}\left(\left(1-q^{2}\right) q^{2 M+2}\right) \operatorname{Sin}_{q^{2}}\left(\left(1-q^{2}\right) q^{2 M+4} s\right) \\
+ & \left.\sin _{q^{2}}\left(\left(1-q^{2}\right) q^{2 M+2}\right) \operatorname{Cos}_{q^{2}}\left(\left(1-q^{2}\right) q^{2 M+4} s\right)\right]
\end{aligned}
$$

В силу предложений 2.1 и 2.2 это выражение равно нулю.

Если же $s=1$, то

$$
\begin{aligned}
& \int d_{q^{2}} z e_{q^{2}}\left(-i\left(1-q^{2}\right) z\right) E_{q^{2}}\left(i\left(1-q^{2}\right) q^{2} z\right) \\
& =\left(1-q^{2}\right) \sum_{m=-\infty}^{\infty} q^{2 m}\left[\frac{\left(-i\left(1-q^{2}\right) q^{2 m+2}, q^{2}\right)_{\infty}}{\left(-i\left(1-q^{2}\right) q^{2 m}, q^{2}\right)_{\infty}}+\frac{\left(i\left(1-q^{2}\right) q^{2 m+2}, q^{2}\right)_{\infty}}{\left(i\left(1-q^{2}\right) q^{2 m}, q^{2}\right)_{\infty}}\right] \\
& =\left(1-q^{2}\right) \sum_{m=-\infty}^{\infty} q^{2 m}\left(\frac{1}{1+i\left(1-q^{2}\right) q^{2 m}}+\frac{1}{1-i\left(1-q^{2}\right) q^{2 m}}\right) \\
& =2 \sum_{m=-\infty}^{\infty} \frac{1}{\left(1-q^{2}\right)^{-1} q^{-2 m}+\left(1-q^{2}\right) q^{2 m}}=\frac{2}{1-q^{2}} \Theta_{0}
\end{aligned}
$$

(см. (2.10) и (2.12)).

Точно так же доказьвается следуюшая 
Лемма 5.2. Справедливо равенство

$$
\int E_{q^{2}}\left(-i\left(1-q^{2}\right) z s\right) E_{q^{2}}\left(i\left(1-q^{2}\right) q^{2} s\right) d_{q^{2}} s= \begin{cases}\frac{2}{1-q^{2}} \Theta_{0} & \text { nрu } z=1, \\ 0 & \text { nрu } z \neq 1\end{cases}
$$

ЛЕмма 5.3. Имеют место соотношения

$$
\begin{aligned}
\mathscr{F}_{q} \Lambda & =q^{-2} \Lambda^{-1} \mathscr{F}_{q^{2}}, & \mathscr{F}_{q^{2}} \partial_{z}=-i s \mathscr{F}_{q^{2}}, & \mathscr{F}_{q^{2}} z=-i q^{-2} \Lambda^{-1} \partial_{s} \mathscr{F}_{q^{2}}, \\
\mathscr{F}_{q^{2}}^{-1} \Lambda & =q^{-2} \Lambda^{-1} \mathscr{F}_{q^{2}}^{-1}, & \mathscr{F}_{q^{2}}^{-1} \partial_{s}=i \Lambda^{-1} \tilde{F}_{q^{2}}^{-1}, & \mathscr{F}_{q^{2}}^{-1} s=i \partial_{z} \mathscr{F}_{q^{2}}^{-1}
\end{aligned}
$$

ДокАЗАТЕЛЬСтво. Нетрудно проверить, что ядра $q^{2}$-интегральных преобразований (5.1) и (5.3) удовлетворяют условиям:

$$
\begin{gathered}
{ }_{0} \Phi_{1}\left(-; 0 ; q^{2}, i\left(1-q^{2}\right) q^{2} z s\right)=\ddagger E_{q^{2}}\left(i\left(1-q^{2}\right) q^{2} z s\right) \ddagger \\
E_{q^{2}}\left(-i\left(1-q^{2}\right) z s\right)=\ddagger e_{q^{2}}\left(i\left(1-q^{2}\right) z s\right) \ddagger
\end{gathered}
$$

и

$$
\begin{aligned}
\partial_{z} \ddagger E_{q^{2}}\left(\left(1-q^{2}\right) a z s\right) \ddagger & =a \ddagger E_{q^{2}}\left(\left(1-q^{2}\right) a q^{2} z s\right) \ddagger s, \\
\partial_{s} \ddagger E_{q^{2}}\left(\left(1-q^{2}\right) a z s\right) \ddagger & =a z \ddagger E_{q^{2}}\left(\left(1-q^{2}\right) a q^{2} z s\right) \ddagger, \\
\partial_{z} \ddagger e_{q^{2}}\left(\left(1-q^{2}\right) a z s\right) \ddagger & =a \ddagger e_{q^{2}}\left(\left(1-q^{2}\right) a z s\right) \ddagger s, \\
\partial_{s} \ddagger e_{q^{2}}\left(\left(1-q^{2}\right) a z s\right) \ddagger & =a z \ddagger e_{q^{2}}\left(\left(1-q^{2}\right) a z s\right) \ddagger .
\end{aligned}
$$

Из (4.5) и последних соотношений мы получаем утверждение леммы.

ДОКАЗАТЕЛЬСТво ПРЕДЛОЖЕНИЯ 5.1. Из формул (5.4) вытекает

$$
\mathscr{F}_{q^{2}} z^{k} \partial_{z}^{l} \phi(z)=(-i)^{k+l} q^{-2 k}\left(\Lambda^{-1} \partial_{s}\right)^{k} s^{l} \mathscr{F}_{q^{2}} \phi(z) .
$$

С другой стороны,

$$
\partial_{s}^{k} s^{l}=(-1)^{k} q^{k(2 l-k+1)} \sum_{j=0}^{k}(-1)^{j} q^{j(j-1)} \frac{\left(q^{-2 l}, q^{2}\right)}{\left(1-q^{2}\right)^{k-j}}\left[\begin{array}{l}
k \\
j
\end{array}\right]_{q^{2}} s^{l-k+j} \partial_{s}^{j} .
$$

Следовательно, если $\phi(z)$ удовлетворяет неравенству (4.1), то ее образ $\mathscr{F}_{q^{2}} \phi(z)$ удовлетворяет неравенству (5.1). Это значит, в частности, что образ сходящейся в $\widehat{S}_{q^{2}}$ последовательности сходится в $\widehat{S}^{q^{2}}$. То же утверждение относительно $\mathscr{F}_{q^{2}}^{-1}$ доказывается аналогично с использованием формул (5.5).

Для доказательства второй части предложения рассмотрим действие операторов Фурье на базисные функции:

$$
\begin{aligned}
\mathscr{F}_{q^{2}} \circ \mathscr{F}_{q^{2}}^{-1} \widehat{\psi}_{n}^{ \pm}(s) & =\widehat{\psi}_{n}^{ \pm}(s), \\
\mathscr{F}_{q^{2}}^{-1} \circ \mathscr{F}_{q^{2}} \widehat{\phi}_{n}^{ \pm}(z) & =\widehat{\phi}_{n}^{ \pm}(z) .
\end{aligned}
$$


Рассмотрим первое соотношение:

$$
\begin{aligned}
\mathscr{F}_{q^{2}} & \circ \mathscr{F}_{q^{2}}^{-1} \widehat{\psi}_{n}^{+}(s) \\
& =\frac{1}{2 \Theta_{0}} \int d_{q^{2}} z\left(\int E_{q^{2}}\left(-i\left(1-q^{2}\right) z \xi\right) \widehat{\psi}_{n}^{+}(\xi) d_{q^{2}} \xi\right){ }_{0} \Phi_{1}\left(-; 0 ; q^{2}, i\left(1-q^{2}\right) q^{2} z s\right) \\
& =\frac{1-q^{2}}{2 \Theta_{0}} q^{2 n} \int d_{q^{2}} z e_{q^{2}}\left(-i\left(1-q^{2}\right) q^{2 n} z\right){ }_{0} \Phi_{1}\left(-; 0 ; q^{2}, i\left(1-q^{2}\right) q^{2} z s\right) \\
& =\frac{1-q^{2}}{2 \Theta_{0}} \int d_{q^{2}} z e_{q^{2}}\left(-i\left(1-q^{2}\right) z\right)_{0} \Phi_{1}\left(-; 0 ; q^{2}, i\left(1-q^{2}\right) q^{-2 n+2} z s\right) .
\end{aligned}
$$

Из леммы 5.1 следует, что

$$
\mathscr{F}_{q^{2}} \circ \mathscr{F}_{q^{2}}^{-1} \widehat{\psi}_{n}^{+}(s)=\left\{\begin{array}{l}
1 \text { при } s=q^{2 n}, \\
0 \text { при } s \neq q^{2 n},
\end{array}\right.
$$

и мы приходим к (5.6). Точно так же, используя лемму 5.2, приходим к (5.7).

\section{§ 6. $q^{2}$-преобразование Фурье $q^{2}$-обобщенных функций}

ОПРЕДЕЛЕНИЕ 6.1. $q^{2}$-преобразование Фурье $q^{2}$-обобщенной функции $f \in \widehat{S}_{q^{2}}^{\prime}$ - это $q^{2}$-обобшенная функция $g \in\left(\widehat{S}^{q^{2}}\right)^{\prime}$, определяемая равенством

$$
\langle g, \psi\rangle=\langle f, \phi\rangle
$$

где $\phi(z)$ - произвольная функция из $S_{q^{2}}$ и $\psi(s) \in \widehat{S}^{q^{2}}-$ ее $q^{2}$-преобразование Фурье.

Предположим, что $q^{2}$-обобшенная функция $f$ отвечает $f(z)$ и $z f(z)$-абсолютно $q^{2}$-интегрируемая функция. Пусть $\phi(z)=\mathscr{F}_{q^{2}}^{-1} \widehat{\psi}(s)$. Тогда

$$
\begin{aligned}
\langle f, \phi\rangle & =\frac{1}{2 \Theta_{0}} \int d_{q^{2}} z \bar{f}(z) \int E_{q^{2}}\left(-i\left(1-q^{2}\right) z s\right) \psi(s) d_{q^{2}} s \\
& =\frac{1}{2 \Theta_{0}} \int \overline{\int d_{q^{2}} z f(z) E_{q^{2}}\left(i\left(1-q^{2}\right) z s\right)} \psi(s) d_{q^{2}} s=\langle g, \psi\rangle .
\end{aligned}
$$

Это означает, что $q^{2}$-обобщенная функция $g$ отвечает функции

$$
g(s)=\frac{1}{2 \Theta_{0}} \int d_{q^{2}} z f(z) E_{q^{2}}\left(i\left(1-q^{2}\right) z s\right) .
$$

Точно так же, если $g$ определяется абсолютно $q^{2}$-интегрируемой функцией $g(s)$ и $\widehat{\psi}(s)=\mathscr{F}_{q^{2}} \widehat{\phi}(z)$, то

$$
\begin{aligned}
& \langle g, \psi\rangle=\iint d_{q^{2}} z \phi(z)_{0} \Phi_{1}\left(-; 0 ; q^{2}, i\left(1-q^{2}\right) q^{2} z s\right) \bar{g}(s) d_{q^{2}} s \\
& =\int d_{q^{2}} z \phi(z) \overline{\int{ }_{0} \Phi_{1}\left(-; 0 ; q^{2},-i\left(1-q^{2}\right) q^{2} z s\right) g(s) d_{q^{2}} s}=\langle f, \phi\rangle,
\end{aligned}
$$

т.е. $f$ отвечает функции

$$
f(z)=\int{ }_{0} \Phi_{1}\left(-; 0 ; q^{2},-i\left(1-q^{2}\right) q^{2} z s\right) g(s) d_{q^{2}} s .
$$

$q^{2}$-преобразование Фурье $q^{2}$-обобщенных функций из $\widehat{S}_{q^{2}}^{\prime}$ мы обозначим через $\mathscr{F}_{q^{2}}^{\prime}$. Следуюшее предложение вытекает из формул $(6.2),(6.3)$ и леммы 5.3. 
ПРЕДЛОЖЕНИЕ 6.1. В пространстве $q^{2}$-обобщенных функиий имеют место коммутационнье соотношения:

$$
\begin{array}{ll}
\mathscr{F}_{q^{2}}{ }^{2} \Lambda=q^{-2} \Lambda^{-1} \mathscr{F}_{q^{2}}^{\prime}, & \left(\mathscr{F}_{q^{2}}^{\prime}\right)^{-1} \Lambda=q^{-2} \Lambda^{-1}\left(\mathscr{F}_{q^{2}}^{\prime}\right)^{-1}, \\
\mathscr{F}_{{ }^{2}}{ }^{2} \partial_{z}=-i \Lambda^{-1}{ }_{s} \mathscr{F}_{q^{2}}^{\prime}, & \left(\mathscr{F}_{q^{2}}^{\prime}\right)^{-1} \partial_{s}=i z\left(\mathscr{F}_{q^{2}}^{\prime}\right)^{-1}, \\
\mathscr{F}_{q^{2}}{ }^{2} z=-i \partial_{s} \mathscr{F}_{q^{2}}^{\prime}, & \left(\mathscr{F}_{q^{2}}^{\prime}\right)^{-1} s=i q^{-2} \Lambda^{-1} \partial_{z}\left(\mathscr{F}_{q^{2}}{ }^{\prime}\right)^{-1} .
\end{array}
$$

Найдем $q^{2}$-преобразования Фурье некоторых $q^{2}$-обобщенных функций.

1. Из формулы (3.7) следует, что

$$
\mathscr{F}_{q^{2}}^{\prime} z^{-1}=\frac{1}{2 \Theta_{0}} \int d_{q^{2}} z z^{-1} E_{q^{2}}\left(i\left(1-q^{2}\right) z s\right)=\frac{i}{\Theta_{0}} \Theta(s) .
$$

Таким образом, как обобщенная функция над $\widehat{S}^{q^{2}}$

$$
\mathscr{F}_{q^{2}}^{\prime} z^{-1}=i \operatorname{sign} s=i\left(\theta_{q^{2}}^{+}-\theta_{q^{2}}^{-}\right) .
$$

2. Из (6.4), (6.5) и (3.7), (4.9) имеем

$$
\mathscr{F}_{q^{2}}{ }^{2} 1=\mathscr{F}_{q^{2}}{ }^{2} z z^{-1}=-i \partial_{s} \mathscr{F}_{q^{2}}{ }^{2} z^{-1}=\partial_{s}\left(\theta_{q^{2}}^{+}-\theta_{q^{2}}^{-}\right)=2 \delta_{q^{2}} .
$$

3. $\quad \mathscr{F}_{q^{2}}^{\prime}\left(\theta_{q^{2}}^{+}-\theta_{q^{2}}^{-}\right)$

$$
\begin{aligned}
& =\frac{1}{2 \Theta_{0}} \int_{0}^{\infty} d_{q^{2}} z E_{q^{2}}\left(i\left(1-q^{2}\right) z s\right)-\frac{1}{2 \Theta_{0}} \int_{-\infty}^{0} d_{q^{2}} z E_{q^{2}}\left(i\left(1-q^{2}\right) z s\right) \\
& =\frac{1-q^{2}}{2 \Theta_{0}} \sum_{m=-\infty}^{\infty} q^{2 m}\left[e_{q^{2}}\left(i\left(1-q^{2}\right) q^{2 m} s\right)-e_{q^{2}}\left(-i\left(1-q^{2}\right) q^{2 m} s\right)\right] \\
& =\frac{i\left(1-q^{2}\right)}{\Theta_{0}} \sum_{m=-\infty}^{\infty} q^{2 m} \sin _{q^{2}}\left(\left(1-q^{2}\right) q^{2 m} s\right) .
\end{aligned}
$$

Воспользовавшись (2.15), получаем

$$
\begin{aligned}
\mathscr{F}_{q^{2}}^{\prime}\left(\theta_{q^{2}}^{+}-\theta_{q^{2}}^{-}\right) & =\frac{i s^{-1}}{\Theta_{0}} \lim _{M \rightarrow \infty}\left(1-q^{2}\right) s \sum_{m=-M}^{\infty} q^{2 m} \sin _{q^{2}}\left(\left(1-q^{2}\right) q^{2 m}\right) \\
& =\frac{i s^{-1}}{\Theta_{0}} \lim _{M \rightarrow \infty}\left[1-\cos _{q^{2}}\left(\left(1-q^{2}\right) q^{2 M}\right)\right]=\frac{i s^{-1}}{\Theta_{0}} .
\end{aligned}
$$

4. Из (4.9), (6.4) и (6.7) выводим

$$
\mathscr{F}_{q^{2}}^{\prime} \delta=\frac{1}{2} \mathscr{F}_{q^{2}}^{\prime} \partial_{z}\left(\theta_{q^{2}}^{+}-\theta_{q^{2}}^{-}\right)=-\frac{i}{2} \Lambda^{-1} s \mathscr{F}_{q^{2}}^{\prime}\left(\theta_{q^{2}}^{+}-\theta_{q^{2}}^{-}\right)=\frac{1}{2 \Theta_{0}} .
$$

5. Из (4.7) и (4.8), (6.6) и (6.7) находим

$$
\begin{aligned}
& \mathscr{F}_{q^{2}}^{\prime} \theta_{q^{2}}^{+}=\frac{1}{2} \mathscr{F}_{q^{2}}^{\prime}\left(\theta_{q^{2}}^{+}-\theta_{q^{2}}^{-}+1\right)=\frac{i s^{-1}}{2 \Theta_{0}}+\delta_{q^{2}}, \\
& \mathscr{F}_{q^{2}}^{\prime} \theta_{q^{2}}^{-}=\frac{1}{2} \mathscr{F}_{q^{2}}^{\prime}\left(-\theta_{q^{2}}^{+}+\theta_{q^{2}}^{-}+1\right)=-\frac{i s^{-1}}{2 \Theta_{0}}+\delta_{q^{2}} .
\end{aligned}
$$


ПРЕДЛОЖЕНИЕ 6.2. Для любого целого $n \geqslant 0$

$$
\mathscr{F}_{q^{2}}^{\prime} z^{n}=2 i^{n} q^{-n(n+1)} \frac{\left(q^{2}, q^{2}\right)_{n}}{\left(1-q^{2}\right)^{n}} s^{-n} \delta_{q^{2}}(s)
$$

ДокАЗАТЕЛЬСТво. Из формулы (3.2) следует, что

$$
1=\frac{\left(1-q^{2}\right)^{n}}{\left(q^{2}, q^{2}\right)_{n}} \partial_{z}^{n} z^{n}
$$

Тогда из (6.6) и предложения 6.1

$$
\delta_{q^{2}}(s)=\frac{1}{2} \mathscr{F}_{q^{2}}^{\prime} 1=\frac{\left(1-q^{2}\right)^{n}}{2\left(q^{2}, q^{2}\right)_{n}} \mathscr{F}_{q^{2}}^{\prime} \partial_{z}^{n} z^{n}=(-i)^{n} \frac{\left(1-q^{2}\right)^{n}}{2\left(q^{2}, q^{2}\right)_{n}}\left(\Lambda^{-1} s\right)^{n} \mathscr{F}_{q^{2}}^{\prime} z^{n}
$$

Отсюда

$$
\mathscr{F}_{q^{2}}^{\prime} z^{n}=2 i^{n} \frac{\left(q^{2}, q^{2}\right)_{n}}{\left(1-q^{2}\right)^{n}}\left(s^{-1} \Lambda\right)^{n} \delta_{q^{2}}(s) .
$$

Так как $\left(s^{-1} \Lambda\right)^{n}=q^{-n(n-1)} s^{-n} \Lambda^{n}$ и $\delta_{q^{2}}\left(q^{2 n} s\right)=q^{-2 n} \delta_{q^{2}}(s)$, то

$$
\left(s^{-1} \Lambda\right)^{n} \delta_{q^{2}}(s)=q^{-n(n+1)} s^{-n} \delta_{q^{2}}(s) .
$$

Отсюда и из (6.8) вытекает утверждение предложения.

ПРЕДЛОЖЕНИЕ 6.3. Для любого иелого $n \geqslant 0$

$$
\mathscr{F}_{q^{2}}^{\prime} z^{-n-1}=i^{n+1} \frac{\left(1-q^{2}\right)^{n}}{\left(q^{2}, q^{2}\right)_{n}} s^{n} \operatorname{sign} s .
$$

ДоКАЗАТЕЛЬСТво. Из (3.3) следует, что для любого целого $n \geqslant 0$

$$
\partial_{z}^{n} z^{-1}=(-1)^{n} q^{-n(n+1)} \frac{\left(q^{2}, q^{2}\right)_{n}}{\left(1-q^{2}\right)^{n}} z^{-n-1}
$$

Используя предложение 6.1, находим

$$
\begin{aligned}
\mathscr{F}_{q^{2}}^{\prime} z^{-n-1} & =(-1)^{n} q^{n(n+1)} \frac{\left(1-q^{2}\right)^{n}}{\left(q^{2}, q^{2}\right)_{n}} \mathscr{F}_{q^{2}}^{\prime} \partial_{z}^{n} z^{-1} \\
& =i^{n} q^{n(n+1)} \frac{\left(1-q^{2}\right)^{n}}{\left(q^{2}, q^{2}\right)_{n}}\left(\Lambda^{-1} s\right)^{n} \mathscr{F}_{q^{2}}^{\prime} z^{-1}
\end{aligned}
$$

Из (4.5) следует, что $\left(\Lambda^{-1} s\right)^{n}=q^{-n(n+1)} s^{n} \Lambda^{-n}$. Используя этот факт и (6.5), получаем утверждение предложения. 
ПРЕДЛОЖЕНИЕ 6.4. Имеют место равенства

$$
\begin{aligned}
& \mathscr{F}_{q^{2}}^{\prime} z_{+}^{\nu-1}=\frac{e_{q^{2}}\left(q^{2}\right) E_{q^{2}}\left(-q^{2(1-\nu)}\right)}{2 \Theta_{0}}\left(\bar{c}_{\nu} s_{-}^{-\nu}+c_{\nu} s_{+}^{-\nu}\right), \\
& \mathscr{F}_{q^{2}}^{\prime} z_{-}^{\nu-1}=-\frac{e_{q^{2}}\left(q^{2}\right) E_{q^{2}}\left(-q^{2(1-\nu)}\right)}{2 \Theta_{0}}\left(c_{\nu} s_{-}^{-\nu}+\bar{c}_{\nu} s_{+}^{-\nu}\right),
\end{aligned}
$$

$2 \partial e$

$$
c_{\nu}=\sum_{m=-\infty}^{\infty} \frac{q^{2 \nu m}\left(q^{-2 m}+i\left(1-q^{2}\right)\right)}{\left(1-q^{2}\right)^{-1} q^{-2 m}+\left(1-q^{2}\right) q^{2 m}} .
$$

ДокАЗАТЕЛЬСТво. Из (4.10) и (6.2) при $0<\operatorname{Re} \nu<1$

$$
\mathscr{F}_{q^{2}} z_{+}^{\nu-1}=\frac{1-q^{2}}{2 \Theta_{0}} \sum_{m=-\infty}^{\infty} q^{2 \nu m} e_{q^{2}}\left(i\left(1-q^{2}\right) q^{2 m} s\right) .
$$

Легко проверить, что функция $z_{+}^{\nu-1}$ удовлетворяет уравнению

$$
z \partial_{z} f(z)=\frac{1-q^{2(\nu-1)}}{1-q^{2}} f(z)
$$

Из предложения 6.1 следует, что после применения $q^{2}$-преобразования Фурье это уравнение принимает вид

$$
-q^{-2} \partial_{s}\left(s g\left(q^{-2} s\right)\right)=\frac{1-q^{2(\nu-1)}}{1-q^{2}} g(s)
$$

Этому уравнению удовлетворяют функции $s_{-}^{-\nu}$ и $s_{+}^{-\nu}$. Поэтому

$$
\mathscr{F}_{q^{2}}^{\prime} z_{+}^{\nu-1}=c_{1} s_{-}^{-\nu}+c_{2} s_{+}^{-\nu}
$$

Подставляя $s=1$ в (6.12), из (2.6), (6.11) и (6.12) получаем

$$
\begin{aligned}
c_{2} & =\frac{1-q^{2}}{2 \Theta_{0}} \sum_{m=-\infty}^{\infty} q^{2 \nu m} e_{q^{2}}\left(i\left(1-q^{2}\right) q^{2 m}\right) \\
& =\frac{1-q^{2}}{2 \Theta_{0}} e_{q^{2}}\left(q^{2}\right) \sum_{m=-\infty}^{\infty} q^{2 \nu m} \sum_{n=0}^{\infty} \frac{(-1)^{n} q^{n(n+1)}}{\left(q^{2}, q^{2}\right)_{n}\left[1-i\left(1-q^{2}\right) q^{2(m+n)}\right]} .
\end{aligned}
$$

При условии $0<\operatorname{Re} \nu<1$ можно поменять порядок суммирования. Тогда

$$
\begin{aligned}
c_{2} & =\frac{1-q^{2}}{2 \Theta_{0}} e_{q^{2}}\left(q^{2}\right) \sum_{n=0}^{\infty} \frac{(-1)^{n} q^{n(n+1)}}{\left(q^{2}, q^{2}\right)_{n}} \sum_{m=-\infty}^{\infty} \frac{q^{2 \nu m}}{1-i\left(1-q^{2}\right) q^{2(m+n)}} \\
& =\frac{e_{q^{2}\left(q^{2}\right)}}{2 \Theta_{0}} \sum_{n=0}^{\infty} \frac{(-1)^{n} q^{n(n-1)} q^{2(1-\nu) n}}{\left(q^{2}, q^{2}\right)_{n}} \sum_{m=-\infty}^{\infty} \frac{q^{2 \nu m}\left(q^{-2 m}+i\left(1-q^{2}\right)\right)}{\left(1-q^{2}\right)^{-1} q^{-2 m}+\left(1-q^{2}\right) q^{2 m}} .
\end{aligned}
$$


Сумму внутреннего ряда обозначим через $c_{\nu}$, тогда

$$
c_{2}=\frac{c_{\nu}}{2 \Theta_{0}} e_{q^{2}}\left(q^{2}\right) E_{q^{2}}\left(-q^{2(1-\nu)}\right)
$$

Положим $s=-1$ в (6.12). Тогда аналогично получим

$$
c_{1}=\frac{\bar{c}_{\nu}}{2 \Theta_{0}} e_{q^{2}}\left(q^{2}\right) E_{q^{2}}\left(-q^{2(1-\nu)}\right)
$$

Подставляя найденные значения констант в (6.12), приходим к (6.9). Для остальных значений $\nu$, не равных целым числам, определим $\mathscr{F}_{q^{2}}^{\prime} z_{+}^{\nu-1}$ как аналитическое продолжение функции (6.9).

$q^{2}$-преобразование Фурье функции $z_{-}^{\nu-1}, \nu \neq 0, \pm 1, \ldots$,

$$
\mathscr{F}_{q^{2}}^{\prime} z_{-}^{\nu-1}=-\frac{1-q^{2}}{2 \Theta_{0}} \sum_{m=-\infty}^{\infty} q^{2 \nu m} e^{q^{2}}\left(-i\left(1-q^{2}\right) q^{2 m} s\right)
$$

отличается от (6.11) лишь знаком и комплексным сопряжением, т.е. мы имеем (6.10).

Полученные выше результаты сведем в таблицу:

\begin{tabular}{|l|l|}
\hline$f(z)$ & $g(s)=\frac{1}{2 \Theta_{0}} \int d_{q^{2}} z f(z) E_{q^{2}}\left(i\left(1-q^{2}\right) z s\right)$ \\
\hline$\delta_{q^{2}}(z)$ & $\frac{1}{2 \Theta_{0}}$ \\
\hline$\theta_{q^{2}}^{+}(z)$ & $\frac{i}{2 \Theta_{0}} s^{-1}+\delta_{q^{2}}(s)$ \\
\hline$\theta_{q^{2}}^{-}(z)$ & $-\frac{i}{2 \Theta_{0}} s^{-1}+\delta_{q^{2}}(s)$ \\
\hline$z^{n}(n=0,1, \ldots)$ & $2 i^{n} q^{-n(n+1)} \frac{\left(q^{2}, q^{2}\right)_{n}}{\left(1-q^{2}\right)^{n}} s^{-n} \delta_{q^{2}}(s)$ \\
\hline$z^{-n-1}(n=0,1, \ldots)$ & $i^{n+1} \frac{\left(1-q^{2}\right)^{n}}{\left(q^{2}, q^{2}\right)_{n}} s^{\operatorname{sign} s}$ \\
\hline$z_{+}^{\nu-1}(\nu \neq 0, \pm 1, \ldots)$ & $\frac{e_{q^{2}}\left(q^{2}\right) E q^{2}\left(-q^{2(1-\nu)}\right)}{2 \Theta_{0}}\left(\bar{c}_{\nu} s_{-}^{-\nu}+c_{\nu} s_{+}^{-\nu}\right)$ \\
\hline$z_{-}^{\nu-1}(\nu \neq 0, \pm 1, \ldots)$ & $-\frac{e_{q^{2}}\left(q^{2}\right) E q^{2}\left(-q^{2(1-\nu)}\right)}{2 \Theta_{0}}\left(c_{\nu} s_{-}^{-\nu}+\bar{c}_{\nu} s_{+}^{-\nu}\right]$ \\
\hline
\end{tabular}




\section{Список литературы}

1. Chryssomalakos Ch., Zumino B. Integrals and Fourier transforms in the quantum plane // Salamfest. 1993. P. 327-346.

2. Kempf A., Majid S. Algebraic $q$-integration and Fourier theory on quantum and braided spaces // J. Math. Phys. 1994. V. 35. № 12. P. 6802-6837.

3. Koornwinder T. H., Swarttouw R. F. On $q$-analogues of the Fourier and Hankel transforms // Trans. Amer. Math. Soc. 1992. V. 333. № 1. P. 445-461.

4. Askey R., Atakishiyev N. M., Suslov S. K. An analog of the Fourier transformation for a $q$-harmonic oscillator // Symmetries in science VI: From the rotation group to quantum algebras. Proceedings of a symposium held in Bregenz, Austria, August 2-7, 1992 / ed. B. Gruber. New York: Plenum Press, 1993. P. 57-63.

5. Rahman M., Suslov S. Singular analogue of the Fourier transformation for the Askey-Wilson polynomials // Preprint CRM-1915, 1993.

6. Askey R., Rahman M., Suslov S. On a general $q$-Fourier transformation with nonsymmetric kernels // Preprint ISSN 0827-3669, 1994.

7. Гельфанд И. М., Шилов Г. Е. Обобщенные функции и действия над ними. Обобщенные функции. Вьп 1. М.: Физматгиз, 1959.

8. Гаспер Джс, Рахман М. Базисные гипергеометрические ряды. М.: Мир, 1993.

Институт теоретической и экспериментальной физики;

Московский государственньй университет путей сообщения

E-mail: olshanet@heron.itep.ru vrogov@cemi.rssi.ru
Поступила в редакцию

03.02.1998 